\title{
Insatisfação com a imagem corporal em adolescentes do sexo feminino de uma cidade de pequeno porte: prevalência e corre- lações
}

\author{
Dissatisfaction with body image among female adolescents in a small \\ Brazilian town: prevalence and correlates
}

Cilene Rebolho Martins ${ }^{1 *}$, Edio Luiz Petroski ${ }^{1}$

ARTIGO ORIGINAL | ORIGINAL ARTICLE

\begin{abstract}
Este estudo buscou identificar a prevalência e os fatores associados à insatisfação com a imagem corporal em adolescentes de uma cidade de pequeno porte. Participaram da pesquisa 144 estudantes do sexo feminino (nove a 20 anos) da cidade de São Bonifácio/SC. Foram obtidos dados referentes às variáveis idade, zona de domicílio, renda familiar mensal e tempo de TV durante a semana e no final de semana. Utilizou-se o BSQ e o EAT-26 para a avaliação da imagem corporal e do risco para transtornos alimentares. O percentual de gordura foi obtido por meio de medidas de dobras cutâneas. A avaliação da maturação sexual foi realizada por meio dos estágios de desenvolvimento de pelos pubianos e mamas e ocorrência da menarca. Aplicou-se a análise de regressão logística $(p<0.05)$. A prevalência de insatisfação com a imagem corporal foi de $26.4 \%$. As adolescentes com risco de transtornos alimentares apresentaram 7,15 vezes mais chance $(\mathrm{RC}=7.15 ; \mathrm{IC} 95 \%=2.67-19.17 ; p=0,0001) \mathrm{de}$ estarem insatisfeitas. Destaca-se a importância do planejamento e implementação de estratégias nas escolas que visem promover uma maior satisfação corporal na adolescência feminina a fim de prevenir transtornos alimentares nesta população.
\end{abstract}

Palavras-chaves: Imagem corporal, Anorexia Nervosa, Bulimia Nervosa, Puberdade, Composição corporal, Adolescente.

ABSTRACT

This study was designed to identify the prevalence of dissatisfaction with body image and factors associated with it among adolescents from a small town in Brazil. A total of 144 female students (nine to 20 years old) from the town of São Bonifácio, SC, took part. Data were collected on the variables age, location of residence, monthly family income and time spent watching TV on weekdays and at the weekend. The BSQ and EAT-26 were used to assess body image and risk of eating disorders. Body fat percentages were calculated from skin fold measurements. Sexual maturity stages were classified on the basis of pubic hair and breast development and menarche. Logistic regression analysis was used to treat data $(p<0.05)$. The prevalence of dissatisfaction with body image was $26.4 \%$. Adolescent females at risk of eating disorders were 7.15 times more likely to be dissatisfied $(\mathrm{RC}=7.15 ; 95 \% \mathrm{CI}=$ 2.67-19.17; $p=0.0001$ ). These results highlight the importance of planning and implementing strategies within schools designed to promote greater body satisfaction among female adolescents, in order to prevent eating disorders in this population.

Keywords: Body image, Anorexia nervosa, Bulimia nervosa, Puberty, Body composition, Adolescent.

\footnotetext{
Artigo recebido a 09.07.2014; Aceite a 26.03.2014

${ }^{1}$ Universidade Federal de Santa Catarina, Florianópolis, Brasil

* Autor correspondente: Núcleo de Pesquisa em Cineantropometria e Desempenho Humano, Centro de Desportos, Universidade Federal de Santa Catarina, Campus Universitário - Bairro Trindade. Caixa Postal 476. CEP: 88040-900. Florianópolis/SC, Brasil. E-mail: cilenerebolho@yahoo.com.br
} 


\section{INTRODUÇÃO}

A insatisfação com a imagem corporal é um problema evidente em adolescentes de diferentes regiões do mundo (Dumith et al., 2012; Mousa, Mashal, Al-Domi, \& Jibril, 2010; Rodríguez \& Cruz, 2008). As constantes transformações relacionadas ao desenvolvimento puberal, somadas à emergência da sexualidade, valorização de sua função social e a dificuldade em estabelecer a própria identidade geram inquietudes $\mathrm{e}$ sofrimento, e por isto, a adolescência é considerada um período crítico para o desenvolvimento da imagem corporal (Braga, Molina, \& Figueiredo, 2010; Campagna \& de Souza, 2006).

A pressão social para atingir o padrão de beleza atual exerce forte influência na forma como os indivíduos percebem e avaliam o seu corpo (Jankauskiene \& Kardelis, 2005). O desejo de atender a este padrão e a impossibilidade de torná-lo real criam um ambiente de intensa insatisfação com a imagem corporal (Stice \& Whitenton, 2002). Neste contexto, adolescentes do sexo feminino frequentemente adotam práticas inadequadas para perda de peso que podem acarretar no desenvolvimento de transtornos alimentares, como a anorexia e a bulimia (Espinoza, Penelo, \& Raich, 2010).

Estudos realizados com adolescentes brasileiras tem identificado prevalências de insatisfação pelo excesso de peso em torno de $18 \%$ em grandes centros urbanos como São Paulo/SP (Branco, Hilário, \& Cintra, 2006) e Florianópolis/SC (Alves, Vasconcelos, Calvo, \& Neves, 2008). Em cidades de pequeno porte, as prevalências relatadas são, geralmente, maiores, variando de $11,5 \%$ a $71,7 \%$ nos Estados de Minas Gerais (Miranda, Conti, Bastos, \& Ferreira, 2011), Rio Grande do Sul (Corseuil, Pelegrini, Beck, \& Petroski, 2009) e Santa Catarina (Petroski, Pelegrini, \& Glaner, 2012). Estes dados indicam que a insatisfação com a imagem corporal também está presente em adolescentes que residem em cidades pequenas.
As associações entre insatisfação com a imagem corporal e variáveis sociodemográficas (del Duca, Garcia, Sousa, Oliveira, \& Nahas, 2010; Lunde, Frisén, \& Hwang, 2007; Triches \& Giugliani, 2007), nível de adiposidade corporal (Petroski, Pelegrini, \& Glaner, 2009), indicadores de maturação sexual (Scherer, Martins, Pelegrini, Matheus, \& Petroski, 2010) e risco de transtornos alimentares (Alves et al., 2008; Espinoza et al., 2010) tem sido demonstradas em alguns estudos. Entretanto, outros autores não constataram estas relações (Dumith et al., 2012; Fidelix, Silva, Pelegrini, da Silva, \& Petroski, 2011; Martins, Pelegrini, Matheus, \& Petroski, 2010; Mousa et al., 2010; Pelegrini, Silva, da Silva, \& Petroski, 2011; Stice \& Whitenton, 2002), demonstrando a necessidade de explorar o tema a fim de promover um melhor entendimento sobre o assunto, principalmente em cidades de pequeno porte, onde as pesquisas sobre este assunto são recentes.

A exposição à televisão influencia negativamente a imagem corporal de adolescentes (Al Sabbah, Vereecken, Abdeen, Coats, \& Maes, 2009), uma vez que representa um importante meio de disseminação dos padrões estéticos atuais. No entanto, no Brasil, as consequências do tempo excessivo de TV para a imagem corporal de adolescentes são pouco investigadas (del Duca et al., 2010), especialmente quando se trata de uma população que vive em uma cidade pequena.

Diante do exposto, o objetivo do presente estudo foi analisar a prevalência de insatisfação com a imagem corporal em adolescentes do sexo feminino de uma cidade de pequeno porte do Estado de Santa Catarina/SC e identificar quais fatores relacionados à características sociodemográficas (idade, zona de domicílio e renda familiar), físicas (percentual de gordura corporal e indicadores de maturação sexual) e comportamentais (tempo de TV e risco para transtornos alimentares) estão associados a este desfecho. Este conhecimento pode auxiliar no planejamento de ações que visem promover melhoras na imagem corporal de adolescentes. 


\section{MÉTODO}

Este estudo foi desenvolvido com base nos dados da pesquisa "Atividade física e estilo de vida: um estudo de três gerações em São Bonifácio-SC". Trata-se de um levantamento epidemiológico transversal, de base escolar, conduzido em 2010, sob a aprovação do Comitê de Ética em pesquisa com Seres Humanos da Universidade Federal de Santa Catarina (UFSC), processo $\mathrm{n}^{\circ} 973 / 2010$. Os participantes do estudo entregaram o termo de consentimento livre e esclarecido assinado pelos responsáveis legais.

\section{Participantes}

O município de São Bonifácio foi selecionado intencionalmente para a realização da pesquisa utilizando-se como critérios suas características de pequeno porte e colonização germânica, devido à colonização e predominância dessa etnia no estado de Santa Catarina (Governo de Santa Catarina, 2002). Localiza-se a $70 \mathrm{~km}$ de Florianópolis/SC, possui uma área territorial de $461 \mathrm{~km}^{2}$ e a população é de 3.008 habitantes. A economia é baseada na agricultura, com ênfase no plantio de fumo, olericultura e fabricação de laticínios (Instituto Brasileiro de Geografia e Estatística/IBGE, 2010)e o índice de desenvolvimento humano (IDH) é 0,785 , considerado médio (Programa das Nações Unidas para o Desenvolvimento/PNUD, 2000).

Para a realização deste estudo populacional, foram convidados todos os alunos de Ensino Fundamental ( $5^{\mathrm{a}}$ a $8^{\mathrm{a}}$ séries) e Médio de todas as escolas públicas do município de São Bonifácio/SC ( $\mathrm{N}=291)$, onde não há nenhuma escola privada. Desta forma, foi realizado um censo escolar do qual fizeram parte escolares de ambos os sexos, distribuídos em cinco escolas da cidade, sendo uma estadual, localizada no centro da cidade, e onde havia o maior número de alunos, e quatro municipais, pertencentes à zona rural. Para o presente estudo, foram utilizados os dados referentes às adolescentes do sexo feminino, uma vez que questionários específicos de imagem corporal e comportamentos alimentares de risco para transtornos alimentares foram respondidos somente pelas meninas. Um total de 149 adolescentes do sexo feminino de nove a 20 anos participaram do estudo, o que representa $95,5 \%$ da população.

\section{Instrumentos}

As variáveis analisadas foram obtidas por meio de questionário autoaplicável e realização de medidas antropométricas. As informações referentes à idade decimal e zona de domicílio foram autorreferidas pelas adolescentes no questionário, sendo utilizadas as datas de nascimento e data da avaliação para o cálculo da idade decimal. A questão referente à renda familiar mensal foi respondida pelos responsáveis das adolescentes a partir de opções de resposta que estabeleciam faixas de valores em reais, estabelecendo-se duas categorias de análise a partir da distribuição da amostra, sendo elas: Renda menor (até R\$ $1.530,00$ ) e renda maior (acima de $\mathrm{R} \$$ $1.530,00)$.

Para a avaliação da imagem corporal, foi utilizado o Body Shape Questionnaire - BSQ (Cooper, Taylor, Cooper, \& Fairbum, 1987), em sua versão traduzida para o português e validada por Di Pietro e Silveira (2009). A adaptação da escala manteve as características da escala original, apresentando boa consistência interna (alfa de Cronbach=0,97). O BSQ é um questionário autoaplicável do tipo escala de Likert, composto por 34 perguntas que pontuam de um a seis. O somatório das questões pode variar de 34 a 204 pontos, sendo que maiores escores indicam maior preocupação com a imagem corporal e maior autodepreciação devido à aparência física, especialmente no sentido de sentir-se com excesso de peso. A partir da pontuação obtida, as estudantes foram classificadas em satisfeitas (pontuação < 111) ou insatisfeitas (pontuação $\geq 111$ ), conforme adotado por Alves, Vasconcelos, Calvo, e Neves (2008).

Os comportamentos de risco para transtornos alimentares foram avaliados por 
meio do Teste de Atitudes Alimentares (EAT26), que foi elaborado por Garner, Olmsted, Bohr, e Garfinkel (1982) e traduzido para o português e validado por Bighetti (2003), para a sua utilização com adolescentes brasileiras do sexo feminino. A versão de Bighetti (2003) apresentou análise fatorial e consistência interna satisfatórias (alfa de Cronbach $=0,82$ ). O EAT-26 é um questionário de autorrelato composto por 26 questões do tipo escala de Likert, com seis opções de resposta que pontuam de zero a três, estabelecendo a mais alta pontuação indicada pela resposta extrema na direção dos transtornos alimentares (anorexia e bulimia). A pontuação final deste questionário pode variar de 0 a 78 pontos e o ponto de corte utilizado foi 21. Desta forma, considera-se que as adolescentes que pontuaram 21 pontos ou mais apresentam risco ao desenvolvimento de transtornos alimentares, e as que pontuaram menos não apresentam risco.

As informações referentes ao tempo excessivo de TV durante a semana e durante o final de semana foram obtidas por meio das questões: "Quanto tempo por dia você permanece assistindo televisão na semana (segunda a sexta-feira)? E no final de semana (sábado e domingo)?". As adolescentes foram orientadas a registrar o tempo médio por dia, em horas e minutos. Considerou-se tempo excessivo de TV duas horas ou mais por dia em ambas as variáveis (American Academy of Pediatrics, 2001).

As medidas das dobras cutâneas (DC) tricipital (TR) e subescapular (SE) foram mensuradas por dois avaliadores treinados que utilizaram os procedimentos descritos por Norton et al. (2005). Foram utilizados adipômetros da marca CESCORFß, com precisão de $0,1 \mathrm{~mm}$ e um lápis dermográfico para a demarcação dos pontos anatômicos. Foram realizadas duas medidas de cada dobra cutânea, considerando-se a média delas, e quando a diferença entre a $1^{\mathrm{a}}$ e $2^{\mathrm{a}}$ medidas foi superior a $5 \%$, foi realizada uma $3^{\mathrm{a}}$ medida (Gore et al., 2005), considerando-se a mediana das três. O percentual de gordura foi calculado por meio das equações de Slaughter et al. (1988) e classificado de acordo com Lohman (1987). Para fins de análise estatística, as categorias foram agrupadas em "Baixo e normal" (categorias "muito baixo", "baixo" e "normal") e "Alto" (categorias "moderadamente alto", "alto" e "muito alto").

Para o controle de qualidade das medidas antropométricas, foi calculado o Erro Técnico de Medida (ETM) intra e interavaliador (Gore et al., 2005), a partir da tomada de medidas em um grupo de 17 adolescentes (10 moças e sete rapazes) que não fizeram parte da amostra do estudo. Este procedimento é recomendado pela International Society for Advancement in Kinanthropometry (ISAK) para a obtenção de dados confiáveis em pesquisas que utilizam a antropometria como ferramenta (Silva, Pelegrini, Pires-Neto, Vieira, \& Petroski, 2011). Os limites dos ETM intra e interavaliador para as medidas de DC foram $3 \%$ e $7 \%$, respectivamente. Estes valores são considerados aceitáveis para antropometrista intermediário/experiente.

A maturação sexual foi avaliada segundo os estágios de desenvolvimento de pelos pubianos e mamas, de acordo com os critérios propostos por Tanner (1962). Foram utilizadas as figuras elaboradas pelo Departamento de Nutrição da Universidade Federal de Santa Catarina (Adami \& Vasconcelos, 2008) a partir das fotografias de Tanner (1962). A indicação dos estágios foi realizada por autoavaliação, em ambiente reservado, após explicação prévia do instrumento por parte de um pesquisador do sexo feminino. A validade deste método foi comprovada em estudo realizado com escolares brasileiros, o qual utilizou a avaliação médica para comparação (Matsudo \& Matsudo, 2008).

A menarca também foi analisada como indicador de maturação sexual, sendo avaliada pelo "status quo" e pelo método retrospectivo (Baxter-Jones, Eisenmann, \& Sherar, 2005). O "status quo" necessita apenas da idade cronológica da menina no dia da investigação e a resposta "sim" ou "não" para a seguinte 
pergunta: "Você já menstruou?". Para aquelas adolescentes que apresentaram a menarca, foi utilizado o método retrospectivo, solicitando o mês e o ano em que ocorreu. Além disso, a lembrança da data da menarca foi avaliada pelas seguintes opções: a) Com Certeza e b) Sem certeza. Para a descrição da idade da menarca, as adolescentes que marcaram o item "b" foram excluídas.

\section{Procedimentos}

A coleta de dados foi realizada em setembro de 2010 na maior escola da cidade em horário de aula dos alunos por uma equipe composta por professores e alunos de graduação e pósgraduação. Os avaliadores foram previamente treinados para a aplicação dos instrumentos a fim de padronizar todos os procedimentos referentes à obtenção dos dados.

\section{Análise estatística}

A análise de regressão logística foi empregada para verificar os fatores associados à insatisfação com a imagem corporal (desfecho do estudo), com estimativa das razões de chance (RC) e respectivos intervalos de confiança (IC95\%) nas análises bruta e ajustada. $\mathrm{Na}$ análise ajustada, foi construído um modelo hierárquico em três níveis. No primeiro nível (mais distal) foram analisadas as variáveis sociodemográficas (idade, zona de domicílio e renda familiar mensal). As variáveis relacionadas às características físicas (percentual de gordura e indicadores de maturação sexual) foram analisadas no segundo nível e as variáveis comportamentais (risco para transtornos alimentares, tempo de TV durante a semana e tempo de TV durante o final de semana) foram incluídas no terceiro nível (mais proximal). As variáveis foram controladas entre si em cada nível do modelo hierárquico e por aquelas dos níveis anteriores que apresentaram $p$ valor $\leq 0,20$. A variável idade foi tratada de forma contínua e as demais de forma categórica. Utilizou-se o SPSS versão 15.0, adotando-se nível de significância de 5\% em todas as análises.

\section{RESULTADOS}

Das 149 participantes da pesquisa, houve uma perda de cinco adolescentes que não responderam o questionário de imagem corporal porque estavam ausentes na escola na ocasião em que o mesmo foi administrado. Assim, participaram da presente análise 144 adolescentes. Destas, algumas apresentaram dados incompletos para as variáveis zona de domicílio $(n=6)$, renda familiar mensal $(n=$ 42), tempo de TV/dia durante a semana ( $n=$ 7 ), tempo de TV/dia no final de semana ( $n=$ 17) e percentual de gordura $(n=6)$, referente às adolescentes que recusaram-se a realizar as medidas de dobras cutâneas.

Tabela 1

Características da amostra em relação à idade, medidas antropométricas, maturação sexual e pontuação do BSQ e EAT-26. São Bonifácio, SC, 2010.

\begin{tabular}{lcccccc}
\hline Variáveis & $n$ & $\begin{array}{c}\text { Valor míni- } \\
\text { mo }\end{array}$ & $\begin{array}{c}\text { Valor máxi- } \\
\text { mo }\end{array}$ & Média & $\begin{array}{c}\text { Desvio- } \\
\text { padrão }\end{array}$ & Mediana \\
\hline Idade (anos) & 144 & 9.42 & 20.75 & 13.46 & 2.35 & 13.29 \\
DC TR (mm) & 138 & 7.00 & 38.90 & 18.31 & 6.78 & 17.35 \\
DC SE (mm) & 138 & 4.10 & 57.50 & 13.90 & 9.05 & 11.20 \\
Percentual de gordura & 138 & 11.89 & 58.68 & 26.39 & 8.93 & 24.84 \\
Idade da menarca (anos) & 85 & 9.60 & 14.75 & 12.57 & 1.11 & 12.73 \\
Pelos pubianos (estágios) & 144 & 1 & 5 & 3.12 & 1.17 & 3 \\
Mamas (estágios) & 144 & 1 & 5 & 3.27 & 1.12 & 3 \\
Pontuação do BSQ & 144 & 35 & 166 & 87.60 & 31.30 & 82.50 \\
Pontuação do EAT-26 & 144 & 0 & 50 & 15.28 & 10.80 & 13.00 \\
\hline
\end{tabular}

Nota: $n=$ amostra; DC = dobra cutânea; TR = tríceps; SE = subescapular; BSQ = Body Shape Questionnaire; EAT-26 =

Teste de atitudes alimentares. 
Na tabela 1 pode-se observar os valores de tendência central e variabilidade das variáveis idade, medidas antropométricas, indicadores de maturação sexual e pontuação dos questionários BSQ e EAT-26.

A tabela 2 apresenta a distribuição de frequências absoluta (n) e relativa (\%) das variáveis estudadas. Verificou-se que uma maior parte das adolescentes reside na zona rural, possui renda familiar mensal de até $\mathrm{R} \$$ $1.530,00$ reais, percentual de gordura normal, são púberes, já tiveram a menarca e passam mais de duas horas por dia em frente à TV durante a semana e no final de semana. Observou-se que a prevalência de insatisfação com a imagem corporal foi $26,4 \%$ e $27,1 \%$ das adolescentes apresentaram risco para transtornos alimentares (tabela 2).

A Tabela 3 apresenta as razões de chance e intervalos de confiança referentes aos modelos bruto e ajustado para insatisfação com a imagem corporal. Na análise bruta, observa-se que as variáveis idade, percentual de gordura e risco para transtornos alimentares apresentaram associação com o desfecho (tabela 3). Quando a análise foi ajustada de acordo com os níveis hierárquicos, somente $\mathrm{o}$ risco para transtornos alimentares permaneceu associado ao desfecho, mostrando que a chance de insatisfação foi 7,15 vezes maior nas adolescentes que apresentaram esse risco (tabela 3).

\section{DISCUSSÃO}

O estudo atual investigou a insatisfação com a imagem corporal e a associação com fatores sociodemográficos, físicos e comportamentais em adolescentes do sexo feminino de uma cidade de pequeno porte de Santa Catarina. Os achados demonstraram que, aproximadamente, uma em cada quatro estudantes do sexo feminino de nove a 20 anos da cidade de São Bonifácio/SC apresentam insatisfação pelo excesso de peso, e este desfecho mostrou-se fortemente associado aos comportamentos de risco para transtornos alimentares.
Ao comparar a prevalência de insatisfação com a imagem corporal identificada no presente estudo $(26,4 \%)$ com outros estudos que utilizaram o BSQ, verifica-se similaridade ao encontrado em uma cidade de porte médio localizada no centro do Estado do Rio Grande Sul (Santa Maria), onde $25,3 \%$ das adolescentes de 11 a 13 anos apresentaram este desfecho (Martins et al., 2010). Da mesma forma, adolescentes residentes na Espanha (porém, de origem latino-americana), de 13 a 17 anos, e na Jordânia (10 a 16 anos) também apresentaram prevalências semelhantes, sendo $23,6 \%$ e $21,2 \%$, respectivamente (Mousa et al., 2010; Rodríguez \& Cruz, 2008).

Outros estudos que utilizaram o BSQ em adolescentes do sexo feminino encontraram menores prevalências de insatisfação com a imagem corporal, sendo de $11,5 \%$ a $18,8 \%$ em adolescentes espanholas de 13 a 17 anos (Rodríguez \& Cruz, 2008), na cidade de Florianópolis/SC (10 a 19 anos) (Alves et al., 2008) e em estudantes de 15 a 19 anos de cidades de pequeno porte do Estado de Minas Gerais (Miranda et al., 2011). Neste sentido, pode-se dizer que a insatisfação com a imagem corporal não está presente somente em grandes centros urbanos, acometendo também adolescentes que vivem em cidades menores. Este dado é corroborado por estudos realizados em cidades de pequeno porte que utilizaram escalas de silhuetas corporais, os quais mostraram que de $25,2 \%$ a $71,7 \%$ das adolescentes desejavam reduzir o tamanho da silhueta (Corseuil et al., 2009; Fidelix et al., 2011; Petroski et al., 2012). Diante disso, pode-se dizer que a magnitude da influência da mídia em relação ao padrão de beleza ideal não difere entre diferentes contextos, afetando adolescentes tanto de cidades grandes quanto pequenas. 
100 | CR Martins, EL Petroski

Tabela 2

Descrição da amostra de estudantes do sexo feminino de nove a 20 anos de escolas públicas de São Bonifácio/SC, 2010.

\begin{tabular}{|c|c|c|}
\hline Variáveis & $n$ & $\%$ \\
\hline \multicolumn{3}{|c|}{ Zona de domicílio $(n=138)$} \\
\hline Rural & 92 & 66.7 \\
\hline Urbana & 46 & 33.3 \\
\hline \multicolumn{3}{|c|}{ Renda familiar mensal $(n=102)$} \\
\hline Até $\mathrm{R} \$ 1.530,00$ & 63 & 61.8 \\
\hline Acima de $1.530,00$ & 39 & 38.2 \\
\hline \multicolumn{3}{|c|}{ Percentual de gordura $(n=138)$} \\
\hline Muito baixo & 01 & 0.7 \\
\hline Baixo & 10 & 7.2 \\
\hline Normal & 67 & 48.6 \\
\hline Moderadamente alto & 28 & 20.3 \\
\hline Alto & 09 & 6.5 \\
\hline Muito alto & 23 & 16.7 \\
\hline \multicolumn{3}{|c|}{ Desenvolvimento de pelos pubianos $(n=144)$} \\
\hline Pré-púbere & 16 & 11.1 \\
\hline Púbere & 115 & 79.9 \\
\hline Pós-púbere & 13 & 9.0 \\
\hline \multicolumn{3}{|c|}{ Desenvolvimento das mamas $(n=144)$} \\
\hline Pré-púbere & 11 & 7.6 \\
\hline Púbere & 115 & 79.9 \\
\hline Pós-púbere & 18 & 12.5 \\
\hline \multicolumn{3}{|l|}{ Menarca $(n=144)$} \\
\hline Não & 59 & 41.0 \\
\hline Sim & 85 & 59.0 \\
\hline \multicolumn{3}{|c|}{ Tempo de TV/dia durante a semana $(n=137)$} \\
\hline$<2 \mathrm{hs} / \mathrm{dia}$ & 37 & 27.0 \\
\hline$\geq 2 \mathrm{hs} /$ dia & 100 & 73.0 \\
\hline \multicolumn{3}{|c|}{ Tempo de TV/dia durante o final de semana ( $n=127)$} \\
\hline$<2 \mathrm{hs} / \mathrm{dia}$ & 29 & 22.8 \\
\hline$\geq 2 \mathrm{hs} / \mathrm{dia}$ & 98 & 77.2 \\
\hline \multicolumn{3}{|l|}{ BSQ $(n=144)$} \\
\hline Satisfeitas & 106 & 73.6 \\
\hline Insatisfeitas & 38 & 26.4 \\
\hline \multicolumn{3}{|l|}{ EAT-26 $(n=144)$} \\
\hline Sem risco de $\mathrm{TA}$ & 105 & 72.9 \\
\hline Risco de TA & 39 & 27.1 \\
\hline
\end{tabular}

Nota: $n=$ amostra; $\mathrm{BSQ}=$ Body Shape Questionnaire; EAT-26 = Teste de atitudes alimentares; TA = transtornos alimentares. 
Tabela 3

Razão de chance bruta e ajustada para insatisfação com a imagem corporal em relação às variáveis sociodemográficas, físicas e comportamentais em adolescentes do sexo feminino. Categoria de referência: satisfeitas. São Bonifácio/SC, 2010.

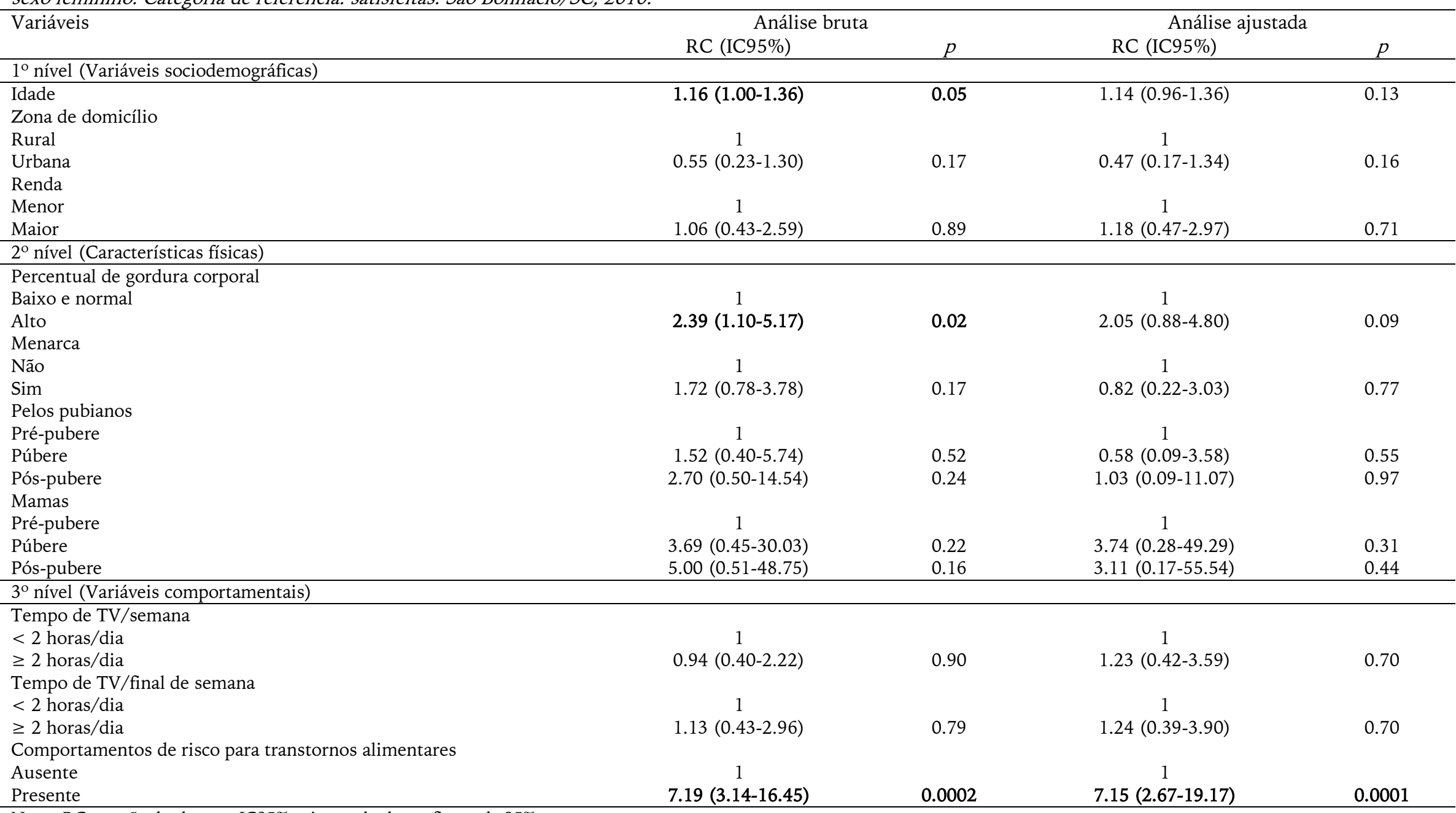

Nota: $R C=$ razão de chance; $I C 95 \%$ = intervalo de confiança de $95 \%$. 
Dentre as variáveis exploratórias analisadas neste estudo, o comportamento de risco para transtornos alimentares apresentou associação com o desfecho. Este resultado foi semelhante ao que tem sido encontrado em outros países, como a Jordânia (Mousa et al., 2010) e Espanha (Espinoza et al., 2010) e na capital de Santa Catarina (Alves et al., 2008). Desta forma, evidencia-se a importância de ações focadas na imagem corporal e na prevenção de transtornos alimentares em adolescentes do sexo feminino, visando uma maior satisfação corporal. O ambiente escolar é um local apropriado para a implementação destas intervenções, pois facilita o alcance e a interação entre um grande número de estudantes em um contexto de aprendizagem, sendo viável tanto em cidades pequenas quanto em centros urbanos maiores e mais desenvolvidos.

Nas escolares de São Bonifácio/SC, a idade não esteve associada à insatisfação com a imagem corporal. Alguns estudos internacionais com delineamento transversal (Mousa et al., 2010) e longitudinal (Lunde et al., 2007) tem demonstrado que o descontentamento em relação à imagem corporal aumenta com a idade em adolescentes do sexo feminino. Entretanto, a maioria dos estudos realizados com adolescentes brasileiras (del Duca et al., 2010; Petroski et al., 2009), inclusive de cidades de pequeno porte (Fidelix et al., 2011; Miranda et al., 2011), são transversais, e mostram que a insatisfação com a imagem corporal não difere em função da idade, corroborando os resultados do presente estudo. Desta forma, considera-se que esta relação deve ser melhor explorada por meio de estudos longitudinais, a fim de verificar se existe uma faixa etária crítica para a insatisfação com a imagem corporal, no sentido de direcionar as intervenções para os grupos de maior risco.

A renda familiar não apresentou associação com o desfecho nas adolescentes de São Bonifácio/SC. Outros estudos conduzidos no Brasil (del Duca et al., 2010; Dumith et al.,
2012) e em outros países (Mousa et al., 2010) não encontraram associação entre nível econômico e insatisfação com a imagem corporal nesta população. Estes resultados mostram que o status socioeconômico não influencia a imagem corporal de adolescentes, indicando que o fato de pertencer a uma classe econômica menos favorecida não significa que não desejam alcançar o padrão de beleza vigente.

No que se refere à área de domicílio, esperava-se que as adolescentes residentes na zona urbana apresentassem maior insatisfação com a imagem corporal, em virtude de uma suposta maior pressão social sobre a forma corporal. Alguns estudos conduzidos em adolescentes de diversas cidades do Estado de Santa Catarina (del Duca et al., 2010) e em escolares de dois municípios de pequeno porte do Rio Grande do Sul confirmam esta hipótese (Triches \& Giugliani, 2007). No entanto, em São Bonifácio/SC, esta relação não foi constatada, corroborando os resultados encontrados em adolescentes de uma cidade de pequeno porte de Minas Gerais (Fidelix et al., 2011) e em municípios do sul do Brasil (Petroski et al., 2009), onde as preocupações em relação à aparência física não diferiram entre adolescentes das zonas rural e urbana. Neste sentido, destaca-se que a zona rural também tem acesso aos meios de comunicação, sendo a TV, muitas vezes, a principal atividade de lazer, e assim, também estão expostos às mesmas influências socioculturais em relação aos padrões de beleza que aqueles que residem na zona urbana. Além disso, em São Bonifácio/SC, adolescentes das áreas rural e urbana convivem na escola e são influenciados pelos pares, destacando-se que, na adolescência, o contexto social é valorizado.

A literatura tem demonstrado que adolescentes do sexo feminino com elevado nível de adiposidade corporal tendem a apresentar maior insatisfação com a imagem corporal (Petroski et al., 2009). Entretanto, no presente estudo, esta relação não se confirmou, bem como nas adolescentes do sexo feminino 
de uma cidade de pequeno porte de Minas Gerais (Pelegrini et al., 2011). Os resultados encontrados em São Bonifácio/SC podem ser explicados pelo tamanho da amostra, que pode não ter sido suficiente para encontrar associação entre o percentual de gordura e a imagem corporal. Outra possível explicação para este achado é que as adolescentes que apresentam nível de gordura considerado adequado também podem estar insatisfeitas.

A relação entre imagem corporal e maturação sexual tem sido pouco explorada na literatura, principalmente a partir da análise do desenvolvimento de pelos pubianos e mamas. O delineamento transversal e o tamanho reduzido da amostra do presente estudo limitaram a interpretação dos resultados referentes a esta questão, sendo necessários estudos longitudinais e com amostras maiores para detectar as mudanças que ocorrem na imagem corporal em função do processo maturacional. Todavia, os resultados encontrados no presente estudo mostraram que não houve associação entre o desfecho e os indicadores de maturação sexual.

Alguns estudos identificaram associação entre menarca e insatisfação com a imagem corporal. Na Jordânia, as adolescentes pósmenarca estavam mais insatisfeitas do que aquelas em que a menarca ainda não havia ocorrido (Mousa et al., 2010). Em escolares da cidade de Santa Maria/RS, verificou-se que a presença da menarca e a sua ocorrência em idades mais precoces fizeram com que as adolescentes apresentassem maior desejo de perder peso (Scherer et al., 2010). Neste sentido, pesquisas futuras devem explorar mais este assunto em adolescentes brasileiras a fim de verificar a influência da maturação na imagem corporal.

O tempo excessivo de TV, tanto durante a semana quanto no final de semana, não se associou à insatisfação corporal no presente estudo. Outros autores têm encontrado resultados semelhantes (del Duca et al., 2010). Esta questão necessita ser mais investigada em função de que a TV é um importante disseminador dos padrões estéticos corporais. Além do tempo de permanência em frente à TV, é importante também analisar quais programas que as adolescentes assistem no sentido de identificar características de conteúdos transmitidos que promovem um aumento nas preocupações com a imagem corporal. Este conhecimento pode auxiliar no desenvolvimento de programas focados na redução da insatisfação com a imagem corporal em adolescentes.

Todavia, ao interpretar os resultados deste estudo, assumimos as seguintes limitações: 1) $\mathrm{O}$ delineamento transversal da pesquisa, que não permite estabelecer uma relação causal entre as variáveis estudadas; 2) Apesar de tratar-se de um estudo populacional, o tamanho da amostra é pequeno, refletindo em uma redução do poder estatístico nas análises; 3) A utilização de questionários, que, por serem instrumentos que avaliam o objeto de estudo de forma subjetiva, e que dependem da capacidade do indivíduo de recordar ações passadas, não garantem a veracidade das respostas fornecidas.

Por outro lado, deve-se considerar que o estudo traz importantes contribuições à literatura, analisando um conjunto de variáveis e a relação com a imagem corporal em adolescentes do sexo feminino de um município de pequeno porte, as quais representam a população de estudantes da cidade. Convém destacar também, a análise de questões pouco exploradas, como a maturação sexual e o tempo excessivo de TV, a utilização de medidas de dobras cutâneas para a avaliação da adiposidade corporal, a inclusão de diferentes marcadores de maturação sexual e a aplicação de questionários validados.

\section{CONCLUSÕES}

Conclui-se que a prevalência de insatisfação com a imagem corporal encontrada no presente estudo foi alta, uma vez que aproximadamente uma em cada quatro adolescentes apresenta este desfecho. A insatisfação foi maior nas adolescentes que apresentaram risco ao 
desenvolvimento de transtornos alimentares. Desta forma, destaca-se a importância do planejamento e implementação de estratégias nas escolas que visem promover uma maior aceitação do corpo na adolescência feminina e uma conscientização a respeito das pressões sociais relacionadas à supervalorização da magreza a fim de prevenir transtornos alimentares nesta população.

Ademais, estudos longitudinais e o uso de metodologias qualitativas se fazem necessários para promover um melhor entendimento deste fenômeno na adolescência.

\section{Agradecimentos:}

Nada a declarar

\section{Conflito de Interesses:}

Nada a declarar.

\section{Financiamento:}

Coordenação de Aperfeiçoamento de Pessoal de Nível Superior (Capes), sob processo número AUXPE PROCAD/NF 110/2010

\section{REFERÊNCIAS}

Adami, F., \& Vasconcelos, F. de A. G. de. (2008). Obesidade e maturação sexual precoce em escolares de Florianópolis - SC. Revista Brasileira de Epidemiologia, 11(4), 549-560. http://doi.org/10.1590/S1415$790 X 2008000400004$

Alves, E., Vasconcelos, F. de A. G. de, Calvo, M. C. M., \& Neves, J. das. (2008). Prevalência de sintomas de anorexia nervosa e insatisfação com a imagem corporal em adolescentes do sexo feminino do Município de Florianópolis, Santa Catarina, Brasil. Cadernos de Saúde Pública, 24(3), 503-512. http://doi.org/10.1590/S0102311X2008000300004

Baxter-Jones, A. D. G., Eisenmann, J. C., \& Sherar, L. B. (2005). Controlling for Maturation in Pediatric Exercise Science. Peditric Exercise Science, $17(1), 18-30$.

Bighetti, F. (2003). Tradução e validação do Eating Attitudes Test (EAT-26) em adolescentes do sexo feminino na cidade de Ribeirão Preto - SP
(Mestrado em Enfermagem). Universidade de São Paulo, Ribeirão Preto.

Braga, P. D., Molina, M. del C. B., \& Figueiredo, T. A. M. de. (2010). Representações do corpo: com a palavra um grupo de adolescentes de classes populares. Ciência \& Saúde Coletiva, 15(1), 87-95. http://doi.org/10.1590/S141381232010000100014

Branco, L. M., Hilário, M. O. E., \& Cintra, I. P. (2006). Percepção e satisfação corporal em adolescentes e a relação com seu estado nutricional. Revista de Psiquiatria Clínica, 33(6), 292-296.

Campagna, V. N., \& de Souza, A. S. L. (2006). Corpo e imagem corporal no início da adolescência feminina. Boletim de Psicologia, 56(124), 935.

Cooper, P. J., Taylor, M. J., Cooper, Z., \& Fairbum, C. G. (1987). The development and validation of the body shape questionnaire. International Journal of Eating Disorders, 6(4), 485-494. http://doi.org/10.1002/1098108X(198707)6:4<485::AIDEAT2260060405>3.0.CO;2-O

Corseuil, M. W., Pelegrini, A., Beck, C., \& Petroski, E. L. (2009). Prevalência de insatisfação com a imagem corporal e sua associação com a inadequação nutricional em adolescentes. Revista da Educação Física/UEM, 20(1), 25-31. http://doi.org/10.4025/reveducfisv20n1p2531

del Duca, G. F., Garcia, L. M. T., Sousa, T. F. de, Oliveira, E. S. A. de, \& Nahas, M. V. (2010). Insatisfação com o peso corporal e fatores associados em adolescentes. Revista Paulista de Pediatria, 28(4), 340-346. http://doi.org/10.1590/S010305822010000400009

Di Pietro, M., \& da Silveira, D. X. (2009). Internal validity, dimensionality and performance of the Body Shape Questionnaire in a group of Brazilian college students. Revista Brasileira de PSiquiatria, $31(1)$, 21-24. http://doi.org/10.1590/S151644462008005000017

Dumith, S. de C., Menezes, A. M. B., Bielemann, R. M., Petresco, S., da Silva, I. C. M., Linhares, R. da S., ... Santos, J. V. dos. (2012). Insatisfação corporal em adolescentes: um estudo de base populacional. Ciência \& Saúde Coletiva, 17(9), 2499-2505. http://doi.org/10.1590/S141381232012000900030

Espinoza, P., Penelo, E., \& Raich, R. M. (2010). Disordered eating behaviors and body image in a longitudinal pilot study of adolescent girls: What happens 2 years later? Body Image, 7(1), 70-73.

http://doi.org/10.1016/j.bodyim.2009.09.002 
Fidelix, Y. L., Silva, D. A. S., Pelegrini, A., da Silva, A. F., \& Petroski, E. L. (2011). Body image dissatisfaction among adolescents from a small town: Association with gender, age, and area of residence. DOI: 10.5007/19800037.2011v13n3p202. Brazilian Journal of Kinanthropometry and Human Performance, 13(3), 202-207. http://doi.org/10.5007/19800037.2011v13n3p202

Garner, D. M., Olmsted, M. P., Bohr, Y., \& Garfinkel, P. E. (1982). The eating attitudes test: psychometric features and clinical correlates. Psychological Medicine, 12(4), 871-878.

Gore, C., Norton, K., Olds, T., Whittingham, N., Birchall, K., Clough, M., ... Downie, L. (2005). Certificação em antropometria: um modelo Australiano. Em K. Norton \& T. Olds (Eds.), Antropométrica: um livro sobre medidas corporais para o esporte e cursos da área de saúde (pp. 375-388). Porto Alegre: ArtMed.

Governo de Santa Catarina. (2002). Colonização de Santa Catarina. Obtido 2 de Dezembro de 2011, www.sc.gov.br/conteudo/santacatarina/histori a/paginas/08imigrantes.html

Instituto Brasileiro de Geografia e Estatística/IBGE. (2010). Censo Populacional 201. Obtido 12 de Dezembro de 2010, de www.ibge.gov.br/home/estatistica/populacao/ censo2010/populacao_por_municipio.shtm

Jankauskiene, R., \& Kardelis, K. (2005). Body image and weight reduction attempts among adolescent girls involved in physical activity. Medicina (Kaunas), 41(9), 796-801.

Lunde, C., Frisén, A., \& Hwang, C. P. (2007). Tenyear-old girls' and boys' body composition and peer victimization experiences: Prospective associations with body satisfaction. Body Image, 4(1),

$11-28$. http://doi.org/10.1016/j.bodyim.2006.10.002

Martins, C. R., Pelegrini, A., Matheus, S. C., \& Petroski, E. L. (2010). Insatisfação com a imagem corporal e relação com estado nutricional, adiposidade corporal e sintomas de anorexia e bulimia em adolescentes. Revista de Psiquiatria do Rio Grande do Sul, 32(1), 19-23. http://doi.org/10.1590/S010181082010000100004

Matsudo, S. M. M., \& Matsudo, V. K. R. (2008). Validade da auto-avaliação na determinação da maturação sexual. Revista Brasileira de Ciência e Movimento, 5(2), 18-35.

Miranda, V. P. N., Conti, M. A., Bastos, R., \& Ferreira, M. E. C. (2011). Insatisfação corporal em adolescentes brasileiros de municípios de pequeno porte de Minas Gerais. Jornal Brasileiro de Psiquiatria, 60(3), 190-197. http://doi.org/10.1590/S004720852011000300007
Mousa, T. Y., Mashal, R. H., Al-Domi, H. A., \& Jibril, M. A. (2010). Body image dissatisfaction among adolescent schoolgirls in Jordan. Body Image, $7(1)$, 46-50. http://doi.org/10.1016/j.bodyim.2009.10.002

Norton, K., Whittingham, N., Carter, L., Kerr, D., Gore, C., \& Marfell-Jones, M. (2005). Técnicas de medição em Antropometria. Em K. Norton \& T. Olds (Eds.), Antropométrica: um livro sobre medidas corporais para o esporte e cursos da área de saúde (pp. 39-87). Porto Alegre: ArtMed.

Pelegrini, A., Silva, D. A. S., da Silva, A. F., \& Petroski, E. L. (2011). Body dissatisfaction among adolescents from a town with a medium/low human development index. Revista Brasileira de Ciências do Esporte, 33(3), 687$698 . \quad$ http://doi.org/10.1590/S010132892011000300011

Petroski, E. L., Pelegrini, A., \& Glaner, M. F. (2009). Insatisfação corporal em adolescentes rurais e urbanos. Motricidade, 5(4), 13-25. http://doi.org/10.6063/motricidade.5(4).167

Petroski, E. L., Pelegrini, A., \& Glaner, M. F. (2012). Reasons and prevalence of body image dissatisfaction in adolescents. Ciência \& Saúde Coletiva, 17(4), 1071-1077. http://doi.org/10.1590/S141381232012000400028

Programa das Nações Unidas para o Desenvolvimento/PNUD. (2000). Ranking do Índice de Desenvolvimento Municipal dos municípios do Brasil. Obtido 20 de Setembro de 2009, de www.pnud.org.br/atlas/tabelas/index.php

Rodríguez, S., \& Cruz, S. (2008). Insatisfacción corporal en adolescentes latinoamericanas y españolas. Psicothema, 20(1), 131-137.

Sabbah, H. Al, Vereecken, C., Abdeen, Z., Coats, E., \& Maes, L. (2009). Associations of overweight and of weight dissatisfaction among Palestinian adolescents: findings from the national study of Palestinian schoolchildren (HBSCWBG2004). Journal of Human Nutrition and Dietetics, 22(1), 40-49. http://doi.org/10.1111/j.1365277X.2008.00901.x

Scherer, F. C., Martins, C. R., Pelegrini, A., Matheus, S. C., \& Petroski, E. L. (2010). Imagem corporal em adolescentes: associação com a maturação sexual e sintomas de transtornos alimentares. Jornal Brasileiro de Psiquiatria, 59(3), 198-202. http://doi.org/10.1590/S004720852010000300005

Silva, D. A. S., Pelegrini, A., Pires-Neto, C. S., Vieira, M. F. S., \& Petroski, E. L. (2011). The anthropometrist in the search for more reliable data. Brazilian Journal of Kinanthropometry and Human Performance, 13(1), 82-85. 
http://doi.org/10.5007/1980-

0037.2011v13n1p82

Stice, E., \& Whitenton, K. (2002). Risk factors for body dissatisfaction in adolescent girls: a longitudinal investigation. Developmental Psychology, 38(5), 669-678.

Tanner, J. M. (1962). Growth at Adolescence. Oxford: Blackwell Scientific Publications.
Triches, R. M., \& Giugliani, E. R. J. (2007). Insatisfação corporal em escolares de dois municípios da região Sul do Brasil. Revista de Nutrição, 20(2),

119-128. http://doi.org/10.1590/S141552732007000200001 quando especificado em contrário e nos conteúdos retirados de outras fontes bibliográficas. 